\title{
Homologous recombination contributes to the repair of DNA double-strand breaks induced by high-energy iron ions
}

Faria Zafar ${ }^{1}$, Sara B. Seidler ${ }^{1}$, Amy Kronenberg, David Schild and Claudia Wiese*

*To whom correspondence should be addressed.

${ }^{1}$ These authors contributed equally to this work.

Lawrence Berkeley National Laboratory, Life Sciences Division, 1 Cyclotron Road, Berkeley, CA 94720, USA.

Tel: $\quad 510-486-4024$

Fax: $\quad 510-486-6816$

Email: cwiese@|lbl.gov

The authors do not have any conflict of interests or financial arrangements to disclose.

Running Title: Homologous recombination repairs DSBs after iron ions

*Work was supported by the U.S. Department of Energy under Contract No. DE-AC02-05CH11231. 


\begin{abstract}
Zafar F., Seidler S.B., Kronenberg A., Schild D. and Wiese C. Homologous recombination contributes to the repair of DNA double-strand breaks induced by high-energy iron ions. Radiat. Res.
\end{abstract}

To test the contribution of homologous recombinational repair (HRR) in repairing DNA damaged sites induced by high-energy iron ions, we used: 1) HRR-deficient rodent cells carrying a deletion in the RAD51D gene and 2) syngeneic human cells impaired for HRR by RAD51D or RAD51 knockdown using RNA interference. We show that in response to iron ions, HRR contributes to cell survival in rodent cells, and that HRR-deficiency abrogates RAD51 foci formation. Complementation of the HRR defect by human RAD51D rescues both enhanced cytotoxicity and RAD51 foci formation. For human cells irradiated with iron ions, cell survival is decreased, and, in p53 mutant cells, the levels of mutagenesis are increased when HRR is impaired. Human cells synchronized in S phase exhibit more pronounced resistance to iron ions as compared with cells in G1 phase, and this increase in radioresistance is diminished by RAD51 knockdown. These results implicate a role for RAD51-mediated DNA repair (i.e. HRR) in removing a fraction of clustered lesions induced by charged particle irradiation. Our results are the first to directly show the requirement for an intact HRR pathway in human cells in ensuring DNA repair and cell survival in response to high-energy high LET radiation. 


\section{Introduction}

One of the major risks associated with long-term manned space travel is the exposure of astronauts to various types of ionizing radiation (IR). IR induces a plethora of DNA lesions among which DNA double-strand breaks (DSBs) are the most toxic. Importantly, if mis-repaired, DSBs can lead to chromosome aberrations $(1,2)$, cell death and mutations $(3,4)$, and the accumulation of mutations resulting from errors in DNA damage repair is an early step in the cancer development process (5). Consequently, organisms strive to faithfully and completely repair damaged DNA to control both cancer risk and genome stability.

When potentially lethal DSBs occur, cells rejoin these DNA ends either by direct nonhomologous end-joining (NHEJ) or by homologous recombinational DNA repair (HRR). These two pathways are distinct biochemically, have different substrate requirements, operate with different kinetics and are used differently throughout the cell cycle (for review see (6)). NHEJ repairs DSBs by re-ligating their two DNA ends and appears to be more efficient than HRR, since many kinds of DSB ends can be re-joined without the requirement of a homologous template. However, NHEJ can result in imprecise rejoining and mutation. In contrast, a homologous template is required for the HRR pathway, which ensures that DSBs are repaired with high fidelity.

The KU70/80 heterodimer initiates NHEJ and forms an open-ring structure, through which a DNA end can be threaded in a sequence-independent manner (7). KU70/80 recruits DNA-PKcs, which together with the KU heterodimer forms the DNA-PK holoenzyme (8). Once bound to a DNA DSB, DNA-PK displays protein Ser/Thr kinase activity, and phosphorylates itself and several substrates, including XRCC4 (9). DNA-PK autophosphorylation facilitates its dissociation from the DNA, thus making the ends available for ligation by the DNA ligase IVIXRCC4/XLF complex. XRCC4 resembles a scaffolding protein, that recruites other NHEJ 
factors to the break (for review see (10)). Biochemical evidence suggests that DNA-PKcs is required for the recruitment of the DNA ligase IVIXRCC4 complex to the chromatin after DNA damage (11). However, it has also been suggested that DNA-PKcs and the DNA ligase IVIXRCC4 complex are independently recruited to the DSB $(12,13)$. In NHEJ, base pairing of DSB ends is essential, since ligation of blunt ends is not efficient (14). In order to optimize base pairing, terminal base degradation may be required, but is not extensive since NHEJ only efficiently joins DSB ends with overhangs of less than 4 bases (15). In contrast, DNA 5'-end resection to generate single-stranded 3'-overhangs is a prerequisite for DSB repair by HRR and is active in S and G2 phases (for review see (16)). These single-stranded DNA overhangs are initially coated with replication protein A (RPA) that subsequently is replaced by the RAD51 recombinase in an exchange reaction facilitated by the activities of RAD52 and of the RAD51 paralogs XRCC2, XRCC3, RAD51B, RAD51C and RAD51D (for review see (17)). RAD51D and XRCC2 form a complex (18-20) that, through its interaction with the Bloom's protein, also was shown to play a role at later stages of recombination (21). Once assembled, the RAD51nucleoprotein filament searches for the homologous template DNA and catalyzes the formation of the joint heteroduplex molecule that now provides the substrate for DNA synthesis around the site of the DSB. Resolution of the complex separates the recombined molecules into intact duplex DNAs.

High LET charged particles deposit large amounts of energy along the ion trajectories, leading to the induction of highly localized DNA damage (22). The organization of the chromatin and the track structure both affect the energy deposition of high atomic number and energy (HZE) particles and lead to more clustered and non-randomly distributed DNA damage than is observed for low LET radiation (23). Complex, non-randomly distributed DNA damage represents a considerable obstacle to efficient repair compared to isolated adducts $(24,25)$, and it has been shown that iron ion-induced clustered lesions prevent efficient KU70/80 binding (26). 
These spatially correlated DSBs rejoin with slower kinetics and to less completeness than DSBs induced by low LET radiation, such as X-rays (27-31). Whereas X-ray-induced DSBs in G1 primarily are repaired by NHEJ, in S and G2 phase HRR plays a critical role (for review see (16)). However, the contribution of HRR-mediated DNA repair of charged particle-induced complex DSBs is less well understood.

In this study, we have used HRR-deficient rodent cells and a collection of human cell lines impaired in HRR due to gene-specific knockdown to test for the contribution of the HRR pathway in repairing DNA damaged sites introduced by high-energy iron ions. In particular, we asked whether compromised HRR would affect cell survival. For human cells, we tested if HRR defects would alter mutation levels in response to iron-ion irradiation, and also investigated whether S phase cells would show enhanced resistance to iron ions.

\section{Methods}

Cells and Culture Conditions

Parental Chinese Hamster Ovary cells (AA8) and their HRR-deficient derivative 51D1 (hereafter: $\operatorname{rad51} d^{-/}$, or rad51d-deficient), generated via targeted inactivation of the AA8 RAD51D genes (32) were kindly provided by Dr. L.H. Thompson. 51D1 cells complemented by human RAD51D were described previously (33). CHO cell lines were maintained as reported earlier (32, 33).

HCA2-hTERT human foreskin fibroblasts cells were a kind gift from Dr. J. Campisi, maintained as previously described $(34,35)$ and were used between 45 and 50 cumulative population doublings in this study. U2OS-T-REx cells were a kind gift from Dr. Eric Campeau and were maintained in DMEM medium supplemented with $10 \%$ Tet-screened FBS, $1 \%$ Lglutamine, $0.5 \%$ penicillin/streptomycin and $5 \mu \mathrm{g} / \mathrm{ml}$ blasticidine $\mathrm{S}$. U2OS-T-REx-shRNA-rad51 cells were obtained by transfection with pTER-hygro encoding RAD51 small hairpin RNA 
(shRNA) and selection for a stable, tightly regulated single clone. To obtain pTER-hygroshRNARAD51, the following oligos were annealed and cloned into unique Bglll and HindIII sites of pTER-hygro:

5'-GATCCCGAAGCTGGATTCCATACTGTTCAAGAGACAGTATGGAATCCAGCTTCTTTTTGGAAA and 5'-AGCTTTTCCAAAAAGAAGCTGGATTCCATACTGTCTCTTGAACAGTATGGAATCCAGCTTCGG.

This shRNA targets the $127-145$ bp region of the RAD51 ORF. A stable clone, tightly regulated for doxycycline-inducible shRNA expression was selected in the presence of both blasticidin S (for $\mathrm{pT}$-REx selection) and hygromycin $\mathrm{B}$ (for pTER selection). Expression of RAD51 shRNA was induced by adding $1 \mu \mathrm{g} / \mathrm{ml}$ doxycycline to the growth medium for 6 days.

Human lymphoblast cell lines TK6 and WTK1 were maintained as previously described (36). TK6 and WTK1 cells were derived from the same donor (37), but differ in p53 status (38-40). Both cell lines carry the same mutated TK allele $(41,42)$, allowing assessment of TK mutant fractions using the remaining TK wild type allele.

Irradiation

Irradiations with $1 \mathrm{GeV} / \mathrm{n}$ iron ions (LET $151 \mathrm{keV} / \mu \mathrm{m})$ were performed at the NASA Space Radiation Laboratory (NSRL) at Brookhaven National Laboratory, Upton, NY. The dose rates in these experiment varied from $0.5 \mathrm{~Gy} / \mathrm{min}$ to $2 \mathrm{~Gy} / \mathrm{min}$. X-ray experiments were performed using $320 \mathrm{kVp} X$ rays filtered through $0.5 \mathrm{~mm}$ copper at dose rates of $0.5 \mathrm{~Gy} / \mathrm{min}$ to $1 \mathrm{~Gy} / \mathrm{min}$. All exposures were carried out at room temperature.

\section{Synchronization}

Synchronization of HCA2-hTERT was carried out essentially as described in (43). Briefly, for G1/G0 synchronization cells were seeded in $10 \%$ serum containing medium for $24 \mathrm{~h}$, followed by a shift into medium with only $0.2 \%$ FBS for $72 \mathrm{~h}$, then medium with $10 \%$ serum was added 
for $4 \mathrm{~h}$ before exposure to iron ions. To synchronize cells at the G1/S border, medium was replaced by medium containing $10 \%$ FBS for $10 \mathrm{~h}$. Then cells were incubated in $2 \mathrm{mM}$ hydroxyurea-containing regular growth medium for $12 \mathrm{~h}$. To release from the G1/S block, cells were washed $3 \mathrm{X}$ in warm PBS and cultured in drug-free medium for 2 to $3 \mathrm{~h}$ before exposure to the ion beam.

U2OS-T-REx-shRNA-rad51 cells were synchronized at the G1/S border by double thymidine block. At $25-30 \%$ confluency, cells were treated with $2 \mathrm{mM}$ thymidine in regular growth medium for $16 \mathrm{~h}$ (without doxycycline) or $18 \mathrm{~h}$ (with doxycycline). Doxycycline treated cells were exposed to thymidine for extended time periods (18 $\mathrm{h}$ vs. $16 \mathrm{~h}$ ) due to their extended cell cycle division time. Thymidine was removed by washing the monolayer $3 \mathrm{X}$ in warm PBS. Fresh medium without thymidine was added for $8 \mathrm{~h}$ to release cells. Following release, regular growth medium with $2 \mathrm{mM}$ thymidine was added for $16 \mathrm{~h}$ (without doxycycline) or $18 \mathrm{~h}$ (with doxycycline). After the second thymidine block, cells were released by washing in PBS and by adding fresh medium. G1 phase cells (generally $70 \% \pm 5 \%$ of the synchronized cell population) were irradiated immediately after release. Cells synchronized in S phase (generally $65 \% \pm 5 \%$ of the synchronized cell population) were irradiated 4 to $5 \mathrm{~h}$ after release.

\section{Survival Studies}

AA8, rad51 ${ }^{-1-}$ and RAD51D complemented cells were plated immediately following the irradiations in serial dilutions at 100 to 10000 cells per T-25 flask (dependent on the dose applied). After 11 days of incubation, colonies were stained in crystal violet solution. The fraction of surviving cells was obtained by the ratio of colonies obtained for a given dose to the number of colonies obtained in the non-irradiated control, taking the plating efficiency into account. Plating efficiencies for AA8 and RAD51D complemented cells usually were between 0.8 and 0.9. Plating efficiencies for rad51 $d^{/-}$cells were $\sim 0.7$, as described previously (32). 
U2OS-T-Rex-shRNA-rad51 cells were plated immediately following the irradiations in serial dilutions at 200 to 60000 cells per T-25 flask (dependent on the dose applied). After 17 days of incubation, colonies were stained in crystal violet solution. Plating efficiencies ranged from 0.30 to 0.45 .

For human lymphoblastoid cell lines, an aliquot of each culture was seeded immediately following the irradiations into 96 -well plates at 1-60 cells/well to determine the surviving fraction. Colonies were counted after 11 days of growth, and the relative fractions of surviving cells were calculated according to standard methods $(44,45)$.

\section{Mutation Analysis}

The mutation frequencies at the autosomal heterozygous TK locus were determined using standard procedures (45). Cells were treated with CHAT (deoxycytidine, hypoxanthine, aminopterine and thymidine) containing medium for $48 \mathrm{~h}$ to eliminate pre-existing TK mutants from the cell population. Cells were then allowed to recover for $24 \mathrm{~h}$ in THC (thymidine, hypoxanthine and deoxycytidine) containing medium before transfections with siRNAs (see below). After the irradiations, cells were kept in standard growth medium for three days to allow for degradation of remaining $T K$ mRNA, and then were plated in the presence of $2 \mu \mathrm{g} / \mathrm{ml}$ trifluorothymidine (TFT) for selection of TK-deficient mutants as described earlier (36). Colonies were scored after 11 days of incubation (i.e. early-arising mutants), re-fed with TFT and scored again after an additional 7 days of incubation (i.e. late-arising mutants). Cells from each culture were also plated at $\sim 1$ cell/well in the absence of TFT for evaluation of the plating efficiencies. The mutant frequency is the ratio of the plating efficiency in the presence of TFT to the plating efficiency obtained without TFT.

\section{Small Interfering RNA and Transfections}

Small interfering RNAs (siRNAs) were obtained from Qiagen (Valencia, CA). The lead siRNA sequences used were r(CACUCUCUGUAAGCAUAUA)d(TT) and r(AAA UCU UCC CGA 
CAGCCAA)d(TT) for RAD51D\#2 and RAD51D\#6, respectively, r(CCGCUAUUACCGUAUCUUA)d(TT) for XRCC4, and r(UUCUCCGAACGUGUCACGU)d(TT) for the validated negative control. A blast search of the human genome data base was carried out to ensure that these sequences would not target other gene transcripts. Transfections of TK6 or WTK1 cells were performed on the day after THC treatment in serum-free RPMI 1640 medium using lipofectamine2000 (Invitrogen, Carlsbad, CA) according to the instructions of the manufacturer. SiRNA/lipofectamine2000 complexes were formed at a $1: 1$ ratio and the concentrations of the siRNAs in transfections were $100 \mathrm{nM}$. Horse serum was added to $10 \% 4$ to $6 \mathrm{~h}$ post addition of the siRNA/lipofectamin2000 complex. Cells were irradiated $72 \mathrm{~h}$ post transfection and knockdown of RAD51D and XRCC4 was assessed by western blot analysis.

Western Blot Analysis and Antibodies

Western blot analysis was carried out according to our standard procedures (33). The following primary antibodies were used: for RAD51D (Novus Biologicals, Littleton, CO), for XRCC4 (kind gift of Dr. S. Yannone), for QM (Santa Cruz Biotechnology, Santa Cruz, CA) and for RAD51 (EMD, Darmstadt, Germany). HRP-conjugated goat anti-rabbit IgG (Jackson ImmunoResearch Laboratories, West Grove, PA) was used as secondary antibody.

Flow Cytometry (FACS) Analysis

A Beckman Coulter EPICS XL flow cytometer (Beckman Coulter, Fullerton, CA) was used for the analysis of cell cycle distributions. This instrument uses an argon-ion air-cooled laser (emission at $488 \mathrm{~nm} / 15 \mathrm{~mW}$ power) and XL Data Acquisition and Display software. Briefly, $10^{6}$ cells were pelleted, washed in cold PBS, re-pelleted and re-suspended in $50 \mu$ PBS. Cells were fixed by adding $10 \mathrm{ml}$ of ice-cold $70 \%$ ethanol/PBS and kept at $4^{\circ} \mathrm{C}$ for at least 2 days. Ethanolfixed cells then were re-pelleted and re-suspended in $5 \mathrm{ml} \mathrm{30 \%} \mathrm{ethanol/PBS} \mathrm{for} 2$ min at $4^{\circ} \mathrm{C}$, re-pelleted and re-suspended in $1 \mathrm{ml} 0.05 \%$ BSA/PBS for $2 \mathrm{~min}$ at $4^{\circ} \mathrm{C}$. Cells were spun down 
again and re-suspended in staining solution $(40 \mu \mathrm{g} / \mathrm{ml}$ RNase $A$ and $50 \mu \mathrm{g} / \mathrm{ml}$ propidium iodide in PBS) before being analyzed at $488 \mathrm{~nm}$ excitation with a $630 \mathrm{~nm}$ long pass filter.

\section{Indirect Immunofluorescence}

Asynchronous $\mathrm{CHO}$ cells were grown on four-well slides. HCA2-hTERT cells were grown on four-well slides and synchronized in $\mathrm{G} 1 / \mathrm{G} 0$ or in $\mathrm{S}$ phase as described above. For exposure to $1 \mathrm{GeV} / \mathrm{n}$ iron ions, chamber slides were positioned at a small angle $\left(<4^{\circ}\right)$ parallel to the particle beam to obtain particle traversals parallel to the monolayer. Cells were fixed in $4 \%$ paraformaldehyde/0.2\% Triton X-100 in PBS at room temperature for 20 min. Cells were then permeabilized with $0.5 \%$ Triton X-100 in PBS for 20 min, washed in PBS, blocked in $2 \%$ BSA in PBS overnight, and incubated with primary antibodies to RAD51 (H-92; Santa Cruz Biotechnology, Santa Cruz, CA; 1:1000; or ab213; Abcam, Cambridge, MA; 1:500), to 53BP1 (BP13; Millipore, Billerica, MA; 1:1000; or A300; Bethyl Laboratories, Montgomery, TX) or cyclin A (H-432; Santa Cruz Biotechnology, Santa Cruz, CA) in $2 \% B S A / P B S$ at $4^{\circ} \mathrm{C}$ overnight. After further washes with PBS, the cells were incubated for $1 \mathrm{~h}$ with Alexa Fluor 488 goat anti-rabbit IgG or Alexa Fluor 594 goat anti-mouse IgG (Invitrogen, Carlsbad, CA; $4 \mu \mathrm{g} / \mathrm{ml}$ ). DNA was stained by DAPI $(50 \mathrm{ng} / \mathrm{ml})$. The slides were viewed at $2000 \times$ magnification in a Zeiss Axioskop microscope.

\section{Statistical Analysis}

Statistical analysis of cell survival data was performed using the results from three independent experiments for each cell line. The logarithms of the surviving fractions were subjected to regression analysis. The model employed for each cell line and condition (high LET or low LET) represents the best fit to the data. For high LET data, the best fits were obtained with linear regression, while for low LET data the best fits were obtained with second order polynomial regression. The slopes of the fitted curves are reported \pm SE. The statistical analysis was performed with the StatView 4.5 statistical package (Abacus Concepts, Berkeley, CA). 
Statistical analysis of mutant fractions was performed on the data from at least three independent experiments and statistical significance was assessed by Student's $t$ test. $P \leq 0.05$ was considered significant and mutation data are presented as means \pm SE. 


\section{Results}

HRR-deficient hamster cells show enhanced sensitivity to the cytotoxic effects of $1 \mathrm{GeV} / \mathrm{n}$ iron ions

Graded doses of $1 \mathrm{GeV} / \mathrm{n}$ iron ions or X-rays were used to determine the surviving fractions of $\mathrm{CHO}$ wild type (AA8) cells and HRR-impaired rad51d-deficient $\mathrm{CHO}$ cells. Rad51d-deficient cells were described previously $(32,33)$. As shown in Fig.1A, rad51d ${ }^{-1}$ cells show enhanced sensitivity to both iron ions and X-rays. At $10 \%$ survival $\left(D_{10}\right)$, rad51 $d^{1-}$ cells are 1.5 - and 1.7 -fold more sensitive to the cytotoxic effects of iron ions and X-rays than their wild type counterparts. The $\alpha$-values of the fitted survival curves obtained after iron-ion exposure are $-0.004( \pm 1.55 \times$ $\left.10^{-4}\right)$ and $-0.007\left( \pm 1.73 \times 10^{-4}\right)$ for AA8 and rad51d-deficient cells, respectively. The slope parameters for $\alpha$ - and $\beta$-values of the fitted survival curves obtained after X-ray exposure are $0.003\left( \pm 1.46 \times 10^{-4}\right)+2.66 \times 10^{-6}\left( \pm 2.30 \times 10^{-7}\right)$ and $-0.004\left( \pm 4.75 \times 10^{-4}\right)+4.632 \times 10^{-6}( \pm$ $7.50 \times 10^{-7}$ ) for AA8 and rad51d-deficient cells, respectively. The relative fractions of surviving cells for both $\mathrm{CHO}$ AA8 and rad51d $d^{-1}$ cells exhibit the expected higher relative biological effectiveness (RBE) of iron ions (RBE values at $D_{10}$ are 2.4 and 2 for wild type and rad51d ${ }^{-1}$ cells, respectively). Ectopic expression of human RAD51D in rad51d-deficient cells, at a level that fully complements for mitomycin C sensitivity (33), also fully complements rad51d-deficient cells for their enhanced sensitivity to X-rays (Fig. 1A, right panel), and nearly complements the sensitivity of rad51 $d^{--}$cells to iron ions (Fig. 1A, left panel; RBE=2.7), as reflected in the $\alpha$-value of the fitted survival curve for rad51 ${ }^{--}$complemented cells after iron-ion exposure (i.e. - 0.005 $\left.\left( \pm 1.44 \times 10^{-4}\right)\right)$. The $\alpha$ - and $\beta$-values of the fitted survival curve for rad51 $d^{-1-}$ complemented cells after X-ray exposure are $-0.003\left( \pm 4.49 \times 10^{-4}\right)+3.03 \times 10^{-6}\left( \pm 7.08 \times 10^{-7}\right)$. In accord with the higher sensitivity of rad51d-deficient cells to iron ions, RAD51 foci formation in these cells is abrogated after iron ions, but 53BP1 foci formation is not affected (Fig. 1B, panels E and F, 
respectively). RAD51 foci formation is re-established by ectopic expression of human RAD51D in rad51d-deficient cells (Fig. 1B, panel G). These results show that RAD51D (i.e. HRR) is required for limiting the cytotoxic effects of iron ions to $\mathrm{CHO}$ cells, and for ensuring RAD51 focus formation in response to iron-ion irradiation.

HRR-deficient human cells show enhanced sensitivity to the cytotoxic effects of $1 \mathrm{GeV} / \mathrm{n}$ iron ions

Transient knockdown of either RAD51D (in HRR) or XRCC4 (in NHEJ) in human lymphoblats was achieved by RNA interference. As shown in Fig. 2A, Western blot analysis confirmed that the siRNAs were effective and reduced the levels of RAD51D and XRCC4 protein in TK6 and WTK1 cells to $\sim 10-20 \%$ of the wild type levels. Protein-depleted TK6 cells (Fig. 2B) and WTK1 cells (Fig. 2C) show enhanced sensitivity to the cytotoxic effects of both iron ions and X-rays. As compared to TK6 cells transfected with the negative control siRNA, RAD51D-depleted TK6 cells are $\sim 1.3$-fold more sensitive to both iron ions and X-rays at $D_{10}$ levels, whereas XRCC4-depleted TK6 cells are $\sim 1.4$-fold more sensitive to iron ions and $\sim 1.6$ fold more sensitive to X-rays compared to control transfected cells. The RBE values $\left(D_{10}\right)$ for iron ions are $\sim 1.2$ for control and RAD51D-depleted TK6 cells, and $\sim 1$ for XRCC4-depleted TK6 cells. In p53 mutant WTK1 cells, knockdown of either RAD51D or XRCC4 also affects cellular sensitivity to both iron ions and X-rays (Fig. 2C). RAD51D- and XRCC4-depleted WTK1 cells are $\sim 1.2$-fold more sensitive $\left(D_{10}\right)$ to iron ions than control transfected cells, and $\sim 1.3-$ and $\sim 1.6$ fold, respectively, more sensitive $\left(D_{30}\right)$ to X-rays. The RBE values $\left(D_{30}\right)$ are $\sim 1.5$ for control transfected, $\sim 1.2$ for RAD51D-depleted and $\sim 1$ for XRCC4-depleted WTK1 cells. These results show that both HRR (as measured by RAD51D depletion) and NHEJ (as measured by XRCC4 depletion) are required for resistance of TK6 and WTK1 cells to $1 \mathrm{GeV} / \mathrm{n}$ Fe ions. However, the effects of protein knockdown (for both RAD51D and XRCC4) on the fraction of surviving cells 
after iron-ion exposure are more pronounced in p53 wild type TK6 cells than in p53 mutant WTK1 cells.

In human cells, the effects of HRR-deficiency on iron ion-induced TK mutagenesis are dependent on p53 status

We next asked whether impairment of HRR, a generally faithful DNA repair pathway, would affect the levels of iron ion-induced mutagenesis in TK6 and WTK1 cells. TK6 and WTK1 cells differ in p53 status (38-40) but were derived from the same donor (37), and each carry a mutated TK allele on the same chromosome $17(41,42)$, allowing assessment of spontaneous and induced mutant fractions using the remaining TK wild type allele. As shown in Fig. 3A, knockdown of RAD51D or XRCC4 did not increase the background mutant fractions in TK6 cells. For TK6 cells with low levels of RAD51D, no increase of iron ion- or X-ray-induced TK mutant fractions was observed for all doses investigated (Fig. 3A, left and right panel, respectively), suggesting that HRR does not limit mutagenesis after X-rays and after lower doses of iron ions in these p53 wild type cells. Notably, after the highest dose of iron-ions tested (1.2 Gy), less TK mutants were recovered from RAD51D-depleted than from control cells, possibly due to the inability of these highly damaged cells to cope with iron-ion induced clustered lesions. In contrast, knockdown of XRCC4 in TK6 cells reduced the fraction of induced TK mutants to approximately $50 \%$ of wild type levels for both iron ions and X-rays (Fig. 3A, left and right panel, respectively), suggesting that in p53 wild type cells NHEJ contributes to mutagenesis after exposure to both high and low LET radiation. Overall, iron ions were more efficient in inducing TK mutants in TK6 cells than X-rays, and the RBE values for TK mutant fractions of control and protein-depleted cells were $\sim 2$.

In p53 mutant WTK1 cells, knockdown of either RAD51D or XRCC4 did not significantly affect TK background mutant factions (Fig. 3B). However, knockdown of RAD51D led to an increase in recoverable TK mutants both after iron ions (1.6- to 2.4-fold, depending on the dose 
delivered) and after X-rays (1.5- to 2.4-fold, depending on the dose delivered). These results suggest that HRR is required to limit mutagenesis after exposure of WTK1 cells to both high and low LET radiation. In contrast to what we observed for TK6 cells, depletion of XRCC4 had no effect on the fractions of recoverable TK mutants from WTK1 cells exposed to iron ions or Xrays. These results suggest that, under wild type conditions, NHEJ (as measured by XRCC4 knockdown) does not contribute to mutagenesis in these p53 mutant cells. Since increased cell killing was seen for XRCC4-depleted compared to control-depleted WTK1 cells (see Fig. 2C), it is unlikely that the extent of XRCC4 protein depletion was insufficient to uncover a phenotype for IR-induced TK mutagenesis in these p53 mutant cells. Iron ions were more efficient in inducing TK mutants in WTK1 cells than X-rays, and the RBE values for TK mutant fractions of control and protein-depleted cells were $\sim 2$.

RAD51 foci formation in response to iron ions is restricted to S/G2 phase

Following X-irradiation, DNA repair by HRR, as indicated by RAD51 foci formation, is restricted to $S$ and $G 2$ phases of the cell cycle $(46,47)$. However, RAD51 foci formation and their dependence on cell cycle stage in response to high LET radiation had not been investigated. To do so, we synchronized HCA2-hTERT immortalized human fibroblasts in G1/G0 or S phase using a published procedure (43). Synchronization was highly efficient and resulted in $90 \pm 5 \%$ of the total cell population in $\mathrm{G} 1 / \mathrm{G} 0$ and $75 \pm 5 \%$ of the total cell population in $S$ phase at the time of exposure to the iron-ion beam (Supplementary Fig. S1A). Iron ioninduced RAD51 foci were detected in S/G2 cells but not in G1 cells (Supplementary Fig. S1B). Within irradiated nuclei, microfocal accumulations (48) of RAD51 were seen in smaller areas, but, overall, they localized to the same regions as the DSB sensor and checkpoint protein 53BP1 (Supplementary Fig. S2A). RAD51 foci resolved within 24 h post 2 Gy $1 \mathrm{GeV} / \mathrm{n}$ iron ions (Supplementary Fig. S2B). 
$S$ phase human cells show resistance to the cytotoxic effects of $1 \mathrm{GeV} / \mathrm{n}$ iron ions

Next, we investigated whether human cells synchronized in $S$ phase would exhibit cellular resistance to $1 \mathrm{GeV} / \mathrm{n}$ iron ions, as compared to $\mathrm{G} 1$ phase cells. To do so, we used the p53 wild type human U2OS osteosarcoma cell line, which can be synchronized efficiently at the G1/S border using double thymidine block. To compromise HRR in these cells, we generated a U2OS derivative (U2OS-T-Rex-shRNA-rad51) in which tetracycline-regulated expression of a RAD51depleting shRNA can be induced (see Methods). As shown in Fig. 4A, knockdown of RAD51 in U2OS cells was obtained efficiently after treatment with doxycycline, and RAD51 protein levels were reduced to $\sim 30 \%$ and $\sim 50 \%$ of the wild type protein levels for cells synchronized in $\mathrm{G} 1$ and in S, respectively (Note: RAD51 is a protein that is essential for cell viability. This most likely accounts for the moderate levels of RAD51 knockdown observed). For irradiations in G1, cells were exposed to iron ions or X-rays immediately following the second thymidine block. For irradiations in $\mathrm{S}$ phase, cells were exposed to iron ions or to X-rays between 5 and $6 \mathrm{~h}$ post release from the second thymidine block (Fig. 4B), and generally $70 \pm 5 \%$ and $65 \pm 5 \%$ of the synchronized cell populations were in $\mathrm{G} 1$ or S phase at the time of the irradiations, respectively. G1 cells showed increased sensitivity to both $1 \mathrm{GeV} / \mathrm{n}$ iron ions (Fig. 4C; 1.6 -fold) and X-rays (Fig. 4D; 1.7-fold) as determined by colony formation assays, and the RBE values $\left(D_{10}\right)$ obtained were 2.6 and 2.5 for cells synchronized in S phase and G1 phase, respectively. Depletion of RAD51 in S phase cells reduced clonogenic survival after iron-ions (Fig. 4C), but not as extensively as after X-rays (Fig. 4D). These results show that in p53 wild type S phase cells RAD51-mediated HRR reduces the cytotoxicity of high-energy iron ions, although too a smaller extent than observed after X-ray exposure, suggesting the operation of additional $\mathbf{S}$ phase-specific DNA repair processes in human cells exposed to high LET radiation. 


\section{Discussion}

Here, we studied whether homologous recombinational DNA repair (HRR) is an important pathway for the repair of spatially correlated complex DSBs such as introduced by $1 \mathrm{GeV} / \mathrm{n}$ iron ions. Our investigation was initiated by several published reports that approached the question indirectly. First, non-homologous end-joining (NHEJ) was progressively inhibited by an increase in the structural complexity of DSBs in vitro (49), suggesting that secondary lesions, proximal to the DSB end and within the footprint of the eukaryotic NHEJ complex, would interfere with the end-joining process. Second, high LET radiation was shown to stimulate HRR in CHO cells (50), and third, a defect in the ATR signaling and checkpoint protein, which decreased the efficiency of HRR, was shown to increase the sensitivity of human cells to $1 \mathrm{GeV} / \mathrm{n}$ iron ions (51). Interestingly, more recent studies, all carried out in rodent cells, now describe a direct role for HRR in DNA repair after high LET radiation, including $1 \mathrm{GeV} / \mathrm{n}$ iron ions $(26,52)$. These studies suggest that bi-stranded clustered lesions are left unrepaired in G1 phase, to be processed by HRR in S phase through restart of broken replication forks by strand invasion. We also had hypothesized that, for a fraction of spatially correlated breaks induced by high-energy iron ions, extensive resection of the DSB ends to generate protruding 3'-overhangs for HRR would be a more feasible way to repair these lesions than NHEJ.

We have shown that $\mathrm{CHO}$ cells deleted for the RAD51D gene (i.e. deficient in HRR) show increased sensitivity to the cytotoxic effects of iron ions compared to wild type cells, and that ectoptic expression of human RAD51D in rad51d-deleted cells returns their sensitivity to iron ions to close to wild type levels (note: although similar, there is no overlap in $\alpha$-values when the surviving fractions for each cell type are analyzed by linear regression). In these experiments, we have chosen a RAD51D-complemented $\mathrm{CHO}$ clone whose protein expression level for human RAD51D is very similar to that of endogenous hamster RAD51D expressed in AA8 wild type $\mathrm{CHO}$ cells, as demonstrated previously (33). Our results showing full complementation for cell survival in response to graded doses of X-rays also support a role for human RAD51D in 
the response to ionizing radiation. We obtain RBE values for iron ions $\left(D_{10}\right)$ of 2.4 and 2 for wild type and rad51d-deficient cells, respectively, that are virtually identical to the RBE values reported by Wang and collaborators (26) for the same high LET radiation type and AA8 and irs1SF cells. Irs1SF cells also are deficient in HRR due to inactivation of the XRCC3 gene (53). Although NHEJ-deficient rodent cells were not included in our study, they were part of the investigation by Wang and collaborators (26), who obtained RBE values $\left(D_{10}\right)$ of $\sim 1$ for $k u 80$ deficient mouse embryonic fibroblasts (MEFs) exposed to $1 \mathrm{GeV} / \mathrm{n}$ iron ions, supporting their additional results for the specific inhibition of the NHEJ pathway after high LET radiation in rodent cells. From our data we infer that HRR is active in rodent cells after exposure to $1 \mathrm{GeV} / \mathrm{n}$ iron ions, and that proper function of this high-fidelity DNA repair pathway is required to fully protect $\mathrm{CHO}$ cells against the cytotoxic effects of iron ions.

RAD51 is the key protein in HRR and forms a filament on single-stranded DNA, a filament that is essential for homology search and strand invasion. Ongoing HRR can be detected as RAD51 focus formation by immunostaining and fluorescence microscopy, and rodent and human cells impaired in HRR due to loss of a RAD51 paralog are impaired in RAD51 foci formation after low LET IR $(32,47)$. As reported here, in response to high LET iron ions rad51ddeficient $\mathrm{CHO}$ cells also were unable to form RAD51 foci, but ectopic expression of human RAD51D in rad51 ${ }^{/-}$cells reverted RAD51 foci formation back to wild type levels, indicative for 1) the causal relationship between cellular sensitivity and ongoing $H R R$, and 2) the functionality of the heterologous human RAD51D protein in $\mathrm{CHO}$ cells deleted for the hamster RAD51D gene. In hTERT-immortalized human fibroblasts exposed to iron ions, RAD51 foci formation is restricted to S/G2 phase and is absent from G1 phase cells (Supplementary Fig. S1), further pointing to the biological relevance of the HRR pathway after high LET radiation. Since RAD51 directly binds to single-stranded DNA, it also accumulates into micro foci after high LET radiation (Supplementary Fig. S2), as demonstrated earlier after laser microirradiation (48). 
Human lymphoblast cells depleted for RAD51D also show enhanced sensitivity to iron ions. However, the effects of RAD51D protein knockdown on cell survival are more pronounced in p53 wild type TK6 cells than in p53 mutant WTK1 cells. The reason for this difference is unclear at this point, but we speculate that mutagenic DNA repair pathways (i.e. NHEJ, single-strand annealing (SSA) or microhomology-mediated end-joining (MMEJ)) may substitute at higher levels in p53 mutant cells, rescuing cytotoxicity in the absence of fully functional HRR. Conversely, since we observe that RAD51D knockdown is more deleterious for iron ion-induced cytotoxicity in TK6 cells, these p53 wild type cells may rely more heavily on faithful HRR, and may not tolerate unfaithful DNA repair events in substitution for HRR. Our results obtained for high and low LET radiation-induced mutagenesis are in support of this speculation, as very different effects of RAD51D knockdown on iron ion-induced mutagenesis are observed when TK6 and WTK1 cells are assessed (see below). Although the RBE values $\left(D_{10}\right)$ obtained for cell death of control and DNA repair protein-depleted cell lines (both TK6 and WTK1 cells) ranged only from $\sim 1-1.5$, it is interesting to note that for both TK6 cells and WTK1 cells the smallest RBE values (i.e. $\sim 1$ ) were obtained for their respective XRCC4-depleted cell populations. This observation is in accord with the recently published report on repair-deficient rodent cells, demonstrating smallest RBEs for NHEJ-deficiency due to the specific inhibition of this DNA repair pathway by $1 \mathrm{GeV} / \mathrm{n}$ iron ions (26).

The inhibition of the HRR pathway via knockdown of RAD51D has different effects on the outcome of mutagenesis at the thymidine kinase (TK) locus in iron ion-exposed TK6 and WTK1 cells. Notably, when expression of RAD51D is reduced, TK mutant fractions are increased significantly in iron ion- and X-irradiated WTK1 cells. In these cells, approximately twice as many TK mutants were recovered from RAD51D-depleted cells than from control-transfected cells at all iron-ion and X-ray doses investigated. These results suggest that in the absence of faithful HRR, unfaithful DNA repair pathways take over, leading to elevated levels of mutagenesis in permissive (i.e. p53 mutant) human cells. Conversely, no such increase in 
mutagenesis was observed in HRR-compromised p53 wild type cells, suggesting that precise HRR is tightly regulated in TK6 cells, does not lead to the induction of TK mutations and cannot be substituted for by error-prone DNA damage repair pathways. However, down-regulation of NHEJ (i.e. XRCC4) in p53 wild type cells limited mutagenesis after both iron ions and X-rays, demonstrating that NHEJ is a mutagenic DNA repair pathway active in TK6 cells after both high and low LET radiation. Unexpectedly, this was not observed for XRCC4-depleted WTK1 cells, for which the levels of iron ion- and X-ray-induced TK mutant fractions remained at those of control-transfected cells. These results indicate that NHEJ does not contribute to mutagenesis in WTK1 cells under wild type repair conditions. This finding suggests that error-prone homology-mediated events, such as SSA, MMEJ (for review see (54)), or PARP-dependent alternative end-joining $(55,56)$ may account for radiation-induced mutagenesis in these p53 mutant cells. It is unlikely that the levels of XRCC4-depletion were insufficient to uncover a phenotype for reduced radiation-induced mutagenesis in WTK1 cells under conditions when NHEJ was impaired, since the same cells demonstrated reduced clonogenic potential after irradiation.

In our previous investigation (36) we have analyzed iron ion-induced TK mutation spectra in both TK6 and WTK1 parental cells and shown that most of the TK mutants induced show loss of heterozygosity, generated by the formation of large deletions ( 1 silent allele remaining). Only in WTK1 cells did we see a significant fraction of mutants induced $(\sim 1 / 3)$ that were also due to interhomolog recombination (i.e. two silent alleles present). In WTK1 cells with diminished HRR due to knockdown of RAD51D, fewer of these types of TK mutants (2 silent alleles) are expected to arise. Instead, it is likely that a higher fraction of TK mutants will be due to deletion events (1 silent allele). In TK6 cells, we expect that depletion of XRCC4, that reduces the number of recoverable TK mutants after low and high LET radiation, will result primarily in loss of deletion-type mutations. Studies to test these expectations are ongoing. 
Our results for X-ray-induced mutagenesis in repair-compromised TK6 cells are very similar to the findings reported by Zhang and collaborators (57). In TK6 cells exposed to $\gamma$-rays, they showed a decrease in TK mutant frequencies after DNA-PKcs (i.e. NHEJ) knockdown, but no change in TK mutant frequencies after RAD54 (i.e. HRR) knockdown. Although in their investigation the effects of high LET radiation on mutagenesis in TK6 cells were not investigated, the effects of both $\gamma$-ray- and iron ion-induced TK mutagenesis in repaircompromised WTK1 cells were studied (57-59). Similar to us, the authors observed increased $\gamma$ ray-induced TK mutant fractions for HRR-impaired (i.e. knockdown of RAD54) WTK1 cells, but dissimilar to us, the authors observed an increase in $\gamma$-ray- and iron ion-induced TK mutant fractions for NHEJ-impaired (i.e. knockdown of DNA-PKcs) WTK1 cells. The reasons for this discrepancy between their findings (WTK1 cells with DNA-PKcs knockdown) and our findings (WTK1 cells with XRCC4 knockdown) are unclear at this point. However, both observations suggest that a deficiency in NHEJ does not reduce radiation-induced mutagenesis in p53 mutant WTK1 cells. These results presumably are due to the fact that other mutagenic DNA repair pathways, such as SSA, MMEJ, alternative end-joining or unfaithful HRR substitute under conditions when both NHEJ is impaired and p53 is non-functional. Furthermore, it is possible that in the absence of DNA-PKcs but in the presence of XRCC4 (57-59), DSBs are processed by a specific repair mechanism that is particularly mutagenic. We speculate that this repair mechanism cannot operate when DNA-PKcs is present and XRCC4 is diminished, the situation investigated in our study.

HRR is restricted to late $S$ and $G 2$ phase of the cell cycle when the sister chromatid is present, and HRR-defective cells show S phase-dependent radiosensitivity (for review see (6)). In repair-proficient human cells, S phase-dependent radioresistance to high-energy iron ions had not been investigated. To the best of our knowledge, our results for a derivative of U2OS cells are the first to show S phase-dependent radioresistance for human cells in response to 
high LET iron-ion irradiation, and our findings are in accord with findings from Blakeley and coworkers (60) studying synchronized human T-1 cells and $425 \mathrm{MeV} / \mathrm{n}$ neon ions. To our surprise, the difference in radioresistance between U2OS cells irradiated in G1 or S phase was almost as pronounced after iron ions as after X-rays (sensitivity factors $\left(D_{10}\right)$ were 1.6 and 1.8, respectively), suggesting that S phase-specific DNA repair pathways (i.e. HRR and SSA) are as important after iron ion exposure as they are after X-rays. Notably, whereas knockdown of

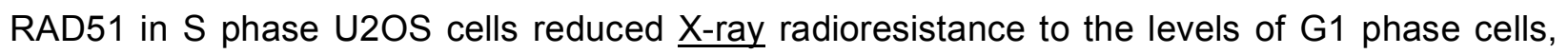
significantly less decrease in radioresistance was observed for iron-ion exposed RAD51depleted cells in S phase. As recently discovered in rodent cells (52), we propose that SSA, a mutagenic sub-pathway of HRR that is restricted to S/G2 cells, and that requires DSB end resection but is RAD51 independent, operates at higher levels after high LET radiation damage than after low LET exposure. However, if we assume that NHEJ is fully functional in $S$ phase U2OS cells depleted for RAD51, it is also possible that in iron ion-irradiated S phase cells a higher fraction of the induced DSBs is repaired by NHEJ. When compared to X-rays, HRR in S phase would then be of less importance after high LET radiation.

In summary, proper HRR is required to fully protect both human and rodent cells from the cytotoxic effects of iron ions. Furthermore, in permissive human cells (i.e. cells with a gain-offunction mutant p53) a reduced level of HRR leads to a significant increase in iron ion-induced mutant fractions. No such increase in mutagenesis is observed in HRR-compromised p53 wild type cells. While HRR is important for some lesion repair (i.e. helps limit cytotoxicity), recombinational repair events in p53 wild type cells are highly controlled, they are mostly faithful, and cannot easily be substituted for by other DSB repair pathways. In addition to HRR, our results support a role for other $S$ phase-specific DNA repair pathways after high LET radiation. These pathways are currently the subject of intense investigation in rodent cells (52), and, in the future, should be investigated in human cells to better understand the astronauts' risk for cancer from space radiation. 


\section{Acknowledgements}

This work was supported by NASA Grant NNJ05HI36I (to CW). The authors gratefully acknowledge Dr. Peter Guida and his group for helpful discussions, guidance and support during beam times, Dr. Adam Rusek and the NSRL physics and dosimetry group for dosimetry and beam delivery and Dr. Betsy Sutherland and her team for assistance. We thank Dr. E. Campeau for parental U2OS-T-REx cells, Dr. S. Yannone for XRCC4 antibody, Dr. L.H. Thompson for AA8 and 51D1 cells, and Dr. J. Campisi for HCA2-hTERT cells. We also would like to thank Hector Nolla (UC Berkeley Flow Cytometry Facility) for his excellent support. 


\section{References}

1. P. E. Bryant, Enzymatic restriction of mammalian cell DNA using Pvu II and Bam H1: evidence for the double-strand break origin of chromosomal aberrations. Int J Radiat Biol Relat Stud Phys Chem Med 46, 57-65 (1984).

2. A. T. Natarajan and G. Obe, Molecular mechanisms involved in the production of chromosomal aberrations. III. Restriction endonucleases. Chromosoma 90, 120-127 (1984).

3. B. Singh and P. E. Bryant, Induction of mutations at the thymidine kinase locus in $\mathrm{CHO}$ cells by restriction endonucleases. Mutagenesis 6, 219-223 (1991).

4. J. W. Phillips and W. F. Morgan, Illegitimate recombination induced by DNA doublestrand breaks in a mammalian chromosome. Mol Cell Biol 14, 5794-5803 (1994).

5. J. B. Little, Radiation carcinogenesis. Carcinogenesis 21, 397-404 (2000).

6. P. Tamulevicius, M. Wang and G. Iliakis, Homology-directed repair is required for the development of radioresistance during S phase: interplay between double-strand break repair and checkpoint response. Radiation Research 167, 1-11 (2007).

7. J. R. Walker, R. A. Corpina and J. Goldberg, Structure of the Ku heterodimer bound to DNA and its implications for double-strand break repair. Nature 412, 607-614 (2001).

8. G. C. Smith and S. P. Jackson, The DNA-dependent protein kinase. Genes \& Development 13, 916-934 (1999).

9. Y. Yu, W. Wang, Q. Ding, R. Ye, D. Chen, D. Merkle, D. Schriemer, K. Meek and S. P. Lees-Miller, DNA-PK phosphorylation sites in XRCC4 are not required for survival after radiation or for V(D)J recombination. DNA Repair 2, 1239-1252 (2003).

10. B. L. Mahaney, K. Meek and S. P. Lees-Miller, Repair of ionizing radiation-induced DNA double-strand breaks by non-homologous end-joining. Biochem J 417, 639-650 (2009).

11. J. Drouet, C. Delteil, J. Lefrancois, P. Concannon, B. Salles and P. Calsou, DNAdependent protein kinase and XRCC4-DNA ligase IV mobilization in the cell in response 
to DNA double strand breaks. The Journal of Biological Chemistry 280, 7060-7069 (2005).

12. P. O. Mari, B. I. Florea, S. P. Persengiev, N. S. Verkaik, H. T. Bruggenwirth, M. Modesti, G. Giglia-Mari, K. Bezstarosti, J. A. Demmers, et al., Dynamic assembly of end-joining complexes requires interaction between Ku70/80 and XRCC4. Proc Natl Acad Sci U S A 103, 18597-18602 (2006).

13. K. Yano and D. J. Chen, Live cell imaging of XLF and XRCC4 reveals a novel view of protein assembly in the non-homologous end-joining pathway. Cell Cycle 7, 1321-1325 (2008).

14. S. J. Boulton and S. P. Jackson, Saccharomyces cerevisiae Ku70 potentiates illegitimate DNA double-strand break repair and serves as a barrier to error-prone DNA repair pathways. EMBO J 15, 5093-5103 (1996).

15. J. M. Daley, P. L. Palmbos, D. Wu and T. E. Wilson, Nonhomologous end joining in yeast. Annual Review of Genetics 39, 431-451 (2005).

16. M. Shrivastav, L. P. De Haro and J. A. Nickoloff, Regulation of DNA double-strand break repair pathway choice. Cell Research 18, 134-147 (2008).

17. J. San Filippo, P. Sung and H. Klein, Mechanism of eukaryotic homologous recombination. Annu Rev Biochem 77, 229-257 (2008).

18. D. Schild, Y. C. Lio, D. W. Collins, T. Tsomondo and D. J. Chen, Evidence for simultaneous protein interactions between human Rad51 paralogs. The Journal of Biological Chemistry 275, 16443-16449 (2000).

19. J. Y. Masson, M. C. Tarsounas, A. Z. Stasiak, A. Stasiak, R. Shah, M. J. Mcllwraith, F. E. Benson and S. C. West, Identification and purification of two distinct complexes containing the five RAD51 paralogs. Genes \& Development 15, 3296-3307 (2001). 
20. C. Wiese, D. W. Collins, J. S. Albala, L. H. Thompson, A. Kronenberg and D. Schild, Interactions involving the Rad51 paralogs Rad51C and XRCC3 in human cells. Nucleic Acids Research 30, 1001-1008 (2002).

21. J. P. Braybrooke, J. L. Li, L. Wu, F. Caple, F. E. Benson and I. D. Hickson, Functional interaction between the Bloom's syndrome helicase and the RAD51 paralog, RAD51L3 (RAD51D). The Journal of Biological Chemistry 278, 48357-48366 (2003).

22. G. Kraft, M. Kramer and M. Scholz, LET, track structure and models. A review. Radiat Environ Biophys 31, 161-180 (1992).

23. B. Rydberg, Radiation-induced DNA damage and chromatin structure. Acta Oncol 40, 682-685 (2001).

24. M. H. David-Cordonnier, J. Laval and P. O'Neill, Clustered DNA damage, influence on damage excision by XRS5 nuclear extracts and Escherichia coli Nth and Fpg proteins. The Journal of Biological Chemistry 275, 11865-11873 (2000).

25. M. H. David-Cordonnier, S. M. Cunniffe, I. D. Hickson and P. O'Neill, Efficiency of incision of an AP site within clustered DNA damage by the major human AP endonuclease. Biochemistry 41, 634-642 (2002).

26. H. Wang, X. Wang, P. Zhang and Y. Wang, The Ku-dependent non-homologous endjoining but not other repair pathway is inhibited by high linear energy transfer ionizing radiation. DNA Repair $7,725-733$ (2008).

27. H. Hoglund and B. Stenerlow, Induction and rejoining of DNA double-strand breaks in normal human skin fibroblasts after exposure to radiation of different linear energy transfer: possible roles of track structure and chromatin organization. Radiation Research 155, 818-825 (2001).

28. K. M. Prise, M. Pinto, H. C. Newman and B. D. Michael, A review of studies of ionizing radiation-induced double-strand break clustering. Radiation Research 156, 572-576 (2001). 
29. M. Pinto, K. M. Prise and B. D. Michael, Evidence for complexity at the nanometer scale of radiation-induced DNA DSBs as a determinant of rejoining kinetics. Radiation Research 164, 73-85 (2005).

30. E. L. Leatherbarrow, J. V. Harper, F. A. Cucinotta and P. O'Neill, Induction and quantification of gamma-H2AX foci following low and high LET-irradiation. International Journal of Radiation Biology 82, 111-118 (2006).

31. A. Asaithamby, N. Uematsu, A. Chatterjee, M. D. Story, S. Burma and D. J. Chen, Repair of HZE-particle-induced DNA double-strand breaks in normal human fibroblasts. Radiation Research 169, 437-446 (2008).

32. J. M. Hinz, R. S. Tebbs, P. F. Wilson, P. B. Nham, E. P. Salazar, H. Nagasawa, S. S. Urbin, J. S. Bedford and L. H. Thompson, Repression of mutagenesis by Rad51Dmediated homologous recombination. Nucleic Acids Research 34, 1358-1368 (2006).

33. C. Wiese, J. M. Hinz, R. S. Tebbs, P. B. Nham, S. S. Urbin, D. W. Collins, L. H. Thompson and D. Schild, Disparate requirements for the Walker A and B ATPase motifs of human RAD51D in homologous recombination. Nucleic Acids Research 34, 28332843 (2006).

34. T. Kumazaki, R. S. Robetorye, S. C. Robetorye and J. R. Smith, Fibronectin expression increases during in vitro cellular senescence: correlation with increased cell area. Experimental Cell Research 195, 13-19 (1991).

35. V. Gorbunova, A. Seluanov and O. M. Pereira-Smith, Expression of human telomerase (hTERT) does not prevent stress-induced senescence in normal human fibroblasts but protects the cells from stress-induced apoptosis and necrosis. The Journal of Biological Chemistry 277, 38540-38549 (2002).

36. C. Wiese, S. S. Gauny, W. C. Liu, C. L. Cherbonnel-Lasserre and A. Kronenberg, Different mechanisms of radiation-induced loss of heterozygosity in two human lymphoid cell lines from a single donor. Cancer Research 61, 1129-1137 (2001). 
37. J. A. Levy, M. Virolainen and V. Defendi, Human lymphoblastoid lines from lymph node and spleen. Cancer 22, 517-524 (1968).

38. J. B. Little, H. Nagasawa, P. C. Keng, Y. Yu and C. Y. Li, Absence of radiation-induced G1 arrest in two closely related human lymphoblast cell lines that differ in p53 status. The Journal of Biological Chemistry 270, 11033-11036 (1995).

39. F. Xia, X. Wang, Y. H. Wang, N. M. Tsang, D. W. Yandell, K. T. Kelsey and H. L. Liber, Altered p53 status correlates with differences in sensitivity to radiation-induced mutation and apoptosis in two closely related human lymphoblast lines. Cancer Research 55, 1215 (1995).

40. W. Zhen, C. M. Denault, K. Loviscek, S. Walter, L. Geng and A. T. Vaughan, The relative radiosensitivity of TK6 and WI-L2-NS lymphoblastoid cells derived from a common source is primarily determined by their p53 mutational status. Mutation Research 346, 85-92 (1995).

41. D. W. Yandell, T. P. Dryja and J. B. Little, Somatic mutations at a heterozygous autosomal locus in human cells occur more frequently by allele loss than by intragenic structural alterations. Somatic Cell and Molecular Genetics 12, 255-263 (1986).

42. J. B. Little and M. B. Benjamin, Molecular structure of mutations at an autosomal locus in human cells: evidence for interallelic homologous recombination. Annales de Genetique 34, 161-166 (1991).

43. A. R. Davalos and J. Campisi, Bloom syndrome cells undergo p53-dependent apoptosis and delayed assembly of BRCA1 and NBS1 repair complexes at stalled replication forks. The Journal of Cell Biology 162, 1197-1209 (2003).

44. E. E. Furth, W. G. Thilly, B. W. Penman, H. L. Liber and W. M. Rand, Quantitative assay for mutation in diploid human lymphoblasts using microtiter plates. Analytical Biochemistry 110, 1-8 (1981). 
45. H. L. Liber and W. G. Thilly, Mutation assay at the thymidine kinase locus in diploid human lymphoblasts. Mutation Research 94, 467-485 (1982).

46. Y. C. Lio, D. Schild, M. A. Brenneman, J. L. Redpath and D. J. Chen, Human Rad51C deficiency destabilizes XRCC3, impairs recombination, and radiosensitizes S/G2-phase cells. The Journal of Biological Chemistry 279, 42313-42320 (2004).

47. C. Wiese, E. Dray, T. Groesser, J. San Filippo, I. Shi, D. W. Collins, M. S. Tsai, G. J. Williams, B. Rydberg, et al., Promotion of homologous recombination and genomic stability by RAD51AP1 via RAD51 recombinase enhancement. Molecular Cell 28, 482490 (2007).

48. S. Bekker-Jensen, C. Lukas, R. Kitagawa, F. Melander, M. B. Kastan, J. Bartek and J. Lukas, Spatial organization of the mammalian genome surveillance machinery in response to DNA strand breaks. The Journal of Cell Biology 173, 195-206 (2006).

49. E. Pastwa, R. D. Neumann, K. Mezhevaya and T. A. Winters, Repair of radiationinduced DNA double-strand breaks is dependent upon radiation quality and the structural complexity of double-strand breaks. Radiation Research 159, 251-261 (2003).

50. G. Olsson, S. Czene, D. Jenssen and M. Harms-Ringdahl, Induction of homologous recombination in the hprt gene of V79 Chinese hamster cells in response to low- and high-LET irradiation. Cytogenetic and Genome Research 104, 227-231 (2004).

51. H. Wang, H. Wang, S. N. Powell, G. lliakis and Y. Wang, ATR affecting cell radiosensitivity is dependent on homologous recombination repair but independent of nonhomologous end joining. Cancer Research 64, 7139-7143 (2004).

52. M. Frankenberg-Schwager, A. Gebauer, C. Koppe, H. Wolf, E. Pralle and D. Frankenberg, Single-strand annealing, conservative homologous recombination, nonhomologous DNA end joining, and the cell cycle-dependent repair of DNA doublestrand breaks induced by sparsely or densely ionizing radiation. Radiation Research 171, 265-273 (2009). 
53. N. Liu, J. E. Lamerdin, R. S. Tebbs, D. Schild, J. D. Tucker, M. R. Shen, K. W. Brookman, M. J. Siciliano, C. A. Walter, et al., XRCC2 and XRCC3, new human Rad51family members, promote chromosome stability and protect against DNA cross-links and other damages. Molecular Cell 1, 783-793 (1998).

54. M. McVey and S. E. Lee, MMEJ repair of double-strand breaks (director's cut): deleted sequences and alternative endings. Trends Genet 24, 529-538 (2008).

55. S. J. DiBiase, Z. C. Zeng, R. Chen, T. Hyslop, W. J. Curran, Jr. and G. Iliakis, DNAdependent protein kinase stimulates an independently active, nonhomologous, endjoining apparatus. Cancer Research 60, 1245-1253 (2000).

56. R. Perrault, H. Wang, M. Wang, B. Rosidi and G. Iliakis, Backup pathways of NHEJ are suppressed by DNA-PK. Journal of Cellular Biochemistry 92, 781-794 (2004).

57. Y. Zhang, J. Zhou, K. D. Held, R. W. Redmond, K. M. Prise and H. L. Liber, Deficiencies of double-strand break repair factors and effects on mutagenesis in directly gammairradiated and medium-mediated bystander human lymphoblastoid cells. Radiation Research 169, 197-206 (2008).

58. Q. Zhang, E. S. Williams, K. F. Askin, Y. Peng, J. S. Bedford, H. L. Liber and S. M. Bailey, Suppression of DNA-PK by RNAi has different quantitative effects on telomere dysfunction and mutagenesis in human lymphoblasts treated with gamma rays or HZE particles. Radiation Research 164, 497-504 (2005).

59. Y. Zhang, J. Zhou, X. Cao, Q. Zhang, C. U. Lim, R. L. Ullrich, S. M. Bailey and H. L. Liber, Partial deficiency of DNA-PKcs increases ionizing radiation-induced mutagenesis and telomere instability in human cells. Cancer Letters 250, 63-73 (2007).

60. E. A. Blakely, P. Y. Chang and L. Lommel, Cell-cycle-dependent recovery from heavyion damage in G1-phase cells. Radiat Res Supp/ 8, S145-157 (1985). 


\section{Figure Legends}

Figure 1. Rad51d-deficient $\mathrm{CHO}$ cells show enhanced sensitivity to the cytotoxic effects of iron ions. 1A: Iron ion- (left panel) and X-ray-induced (right panel) cell killing of wild type, rad51ddeficient and complemented $\mathrm{CHO}$ lines. Data are the means from at least three independent experiments $\pm S D$, and were also fitted by zero-intercept linear regression (Fe ions) and second order polynomial regression (X-rays) using StatView 4.5. The equations for the fitted survival curves are as follows: AA8 (Fe ions): $\log \mathrm{S} / \mathrm{S}_{0}=-0.004\left( \pm 1.55 \times 10^{-4}\right) \times \mathrm{D} ; \mathrm{r}^{2}=0.987$. Rad51 $^{\prime-}$ (Fe ions): $\log \mathrm{S} / \mathrm{S}_{0}=-0.007\left( \pm 1.73 \times 10^{-4}\right) \times \mathrm{D} ; \mathrm{r}^{2}=0.992$. Rad51d ${ }^{-1}+$ RAD51D (Fe ions): $\log$ $S / S_{0}=-0.005\left( \pm 1.44 \times 10^{-4}\right) \times D ; r^{2}=0.992$. AA8 (X-rays): $\log S / S_{0}=0.993-0.003\left( \pm 1.46 \times 10^{-}\right.$ $\left.{ }^{4}\right) \times D+2.659\left( \pm 2.30 \times 10^{-7}\right) \times 10^{-6} D^{2} ; r^{2}=0.991$. Rad51d ${ }^{-1-}(X-$ rays $): \log S / S_{0}=0.959-0.004$ $\left( \pm 4.75 \times 10^{-4}\right) \times D+4.63\left( \pm 7.50 \times 10^{-7}\right) \times 10^{-6} D^{2} ; r^{2}=0.919$. Rad51d ${ }^{-1}+$ RAD51D (X-rays): $\log$ $S / S_{0}=1.002-0.003\left( \pm 4.49 \times 10^{-4}\right) \times D+3.03\left( \pm 7.08 \times 10^{-7}\right) \times 10^{-6} D^{2} ; r^{2}=0.896$. Note: $D=$ dose. 1B: Cytological manifestation of RAD51 nuclear foci in $\mathrm{CHO}$ cells in response to iron ions. Both 53BP1 (panel D) and RAD51 (panel C) form microscopically discernible accumulations as early as $20 \mathrm{~min}$ after exposure of AA8 wild type cells to $1 \mathrm{~Gy}$ of $1 \mathrm{GeV} / \mathrm{n}$ Fe ions. HRR-defective rad51d-knockout cells form 53BP1 foci (panel F) but are unable to form RAD51 foci (panel E) after exposure to iron ions. Ectopic expression of human RAD51D in rad51d-deficient cells restores RAD51 foci formation in response to iron ions (panel G). Panels A \& B: mock-irradiated control cells. Green in panels A, C, E and G: RAD51; green in panels B, D, F and H: 53BP1; blue: DAPI.

Figure 2. Functional loss of RAD51D or XRCC4 sensitizes human lymphoblastoid cells to the cytotoxic effects of iron ions. A: Western blot to show the extent of protein knockdown using the specific siRNAs (as indicated). Neg. control is a non-specific non-depleting siRNA. QM: loading 
control. B: Iron ion- and X-ray-induced cell killing of TK6 cells with RAD51D or XRCC4 knockdown. SiRNA neg. control is a non-specific non-depleting siRNA (see A). C: Iron ion- and X-ray-induced cell killing of WTK1 cells with RAD51D or XRCC4 knockdown. SiRNA neg. control is a non-specific non-depleting siRNA (see A). Data are fitted exponentially and are the means from at least three independent experiments \pm SD.

Figure 3. Iron ion- and X-ray-induced mutant fractions at the thymidine kinase locus in human lymphoblast cell lines after knockdown of RAD51D or XRCC4. A: TK6 cells, B: WTK1 cells. SiRNA neg. control is a non-specific non-depleting siRNA (see Fig. 2A). Data are the means of at least three independent experiments \pm SE. ${ }^{* *} P<0.01,{ }^{*} P<0.05,+P=0.06,0.07,0.08$ and 0.16 (from left to right) compared to cells transfected with the neg. control siRNA. Note: Radiation-induced mutant fractions are corrected for background MF (i.e. MF at 0 Gy).

Figure 4. Synchronization in S phase increases radioresistance of U2OS cells exposed to iron ions. A: Western blot to show the extent of RAD51 knockdown in G1 and S phase U2OS-TRex-shRNA-rad51 cells after treatment with doxycycline. Note: up-regulated levels of RAD51 in untreated S phase cells. B: Cell cycle profiles obtained for U2OS-T-Rex-shRNA-rad51 cells after synchronization by double thymidine block $(\mathrm{G} 1=0 \mathrm{~h} ; \mathrm{S}=5-6 \mathrm{~h}$ post release from second thymidine block (see Methods)). C: Iron ion-induced cell killing of U2OS-T-Rex-shRNA-rad51 cells synchronized in G1 or S phase without and with RAD51 knockdown (i.e. doxy treated). Data are fitted exponentially and are the means from at least three independent experiments \pm SD. D: X-ray-induced cell killing of U2OS-T-Rex-shRNA-rad51 cells synchronized in G1 or S phase without and with RAD51 knockdown (i.e. doxy treated). Data are fitted exponentially and data with error bars the means from at least three independent experiments \pm SD. Data without error bars (G1+doxy, S+doxy) are the means from 2 independent experiments. 
Supplementary Figure S1. RAD51 foci formation in response to iron ions occurs in S/G2 but not in G1 phase cells. S1A: Cell cycle profiles obtained for human HCA2-hTERT immortalized fibroblasts synchronized in G1/G0 (upper right) or in S phase (lower left and right) as described under Methods. S1B: Merged images to show that RAD51 foci formation (red) in response to iron ions occurs in cyclin A-positive cells (green; = S and early G2 phase cells) and not in cyclin A-negative cells (not green: G1 phase cells; arrow in C). Note: panels A and C are identical except for outlined G1 nucleus (based on DAPI staining) in C. DAPI: blue. Non-irradiated cyclin A-positive cells (green in panel D) show elevated, but randomly distributed RAD51 (red in panel D), since the RAD51 protein is up-regulated in S phase. Panels A-C: Cells were fixed at $1 \mathrm{~h}$ post $1.5 \mathrm{~Gy}$ iron ions. Panels D \& E: unirradiated control cells fixed at the same time as cells in panels A-C.

Supplementary Figure S2. RAD51 and 53BP1 localize to the same areas in HCA2-hTERT fibroblasts exposed to iron ions. S1A: Overlap (yellow) between RAD51 (green) and 53BP1 (red) is rare at $30 \mathrm{~min}$ post $2 \mathrm{~Gy} 1 \mathrm{GeV} / \mathrm{n}$ iron ions, but clear at $1 \mathrm{~h}$ post irradiation and persists up to $8 \mathrm{~h}$ post irradiation. S1B: Time course of RAD51 foci formation and resolution in HCA2hTERT fibroblasts exposed to $2 \mathrm{~Gy} 1 \mathrm{GeV} / \mathrm{n}$ iron ions in S phase. Data are the means from three different experiments with 100 nuclei analyzed for each experiment \pm SE. 
Figure 1

A
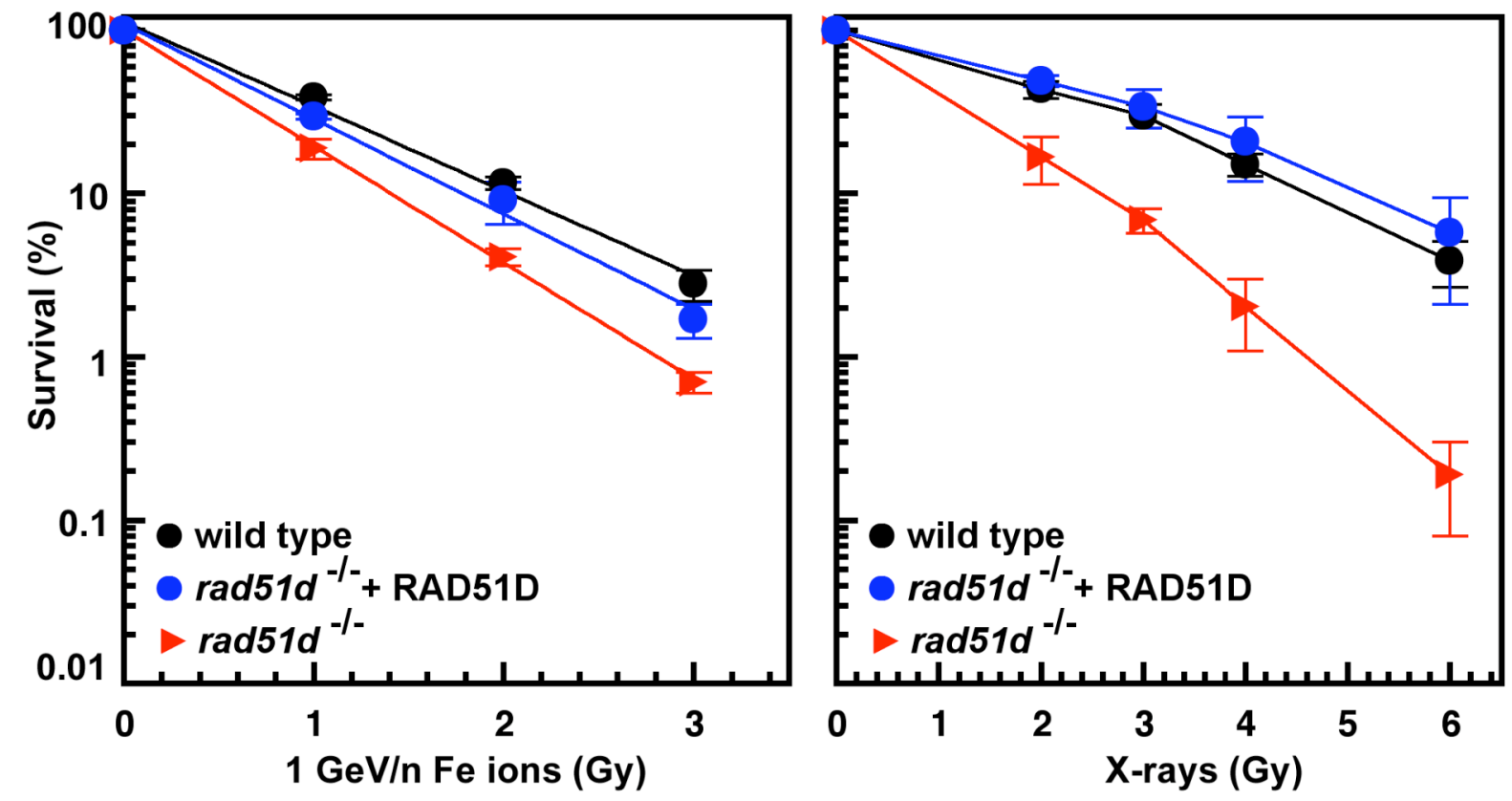

B

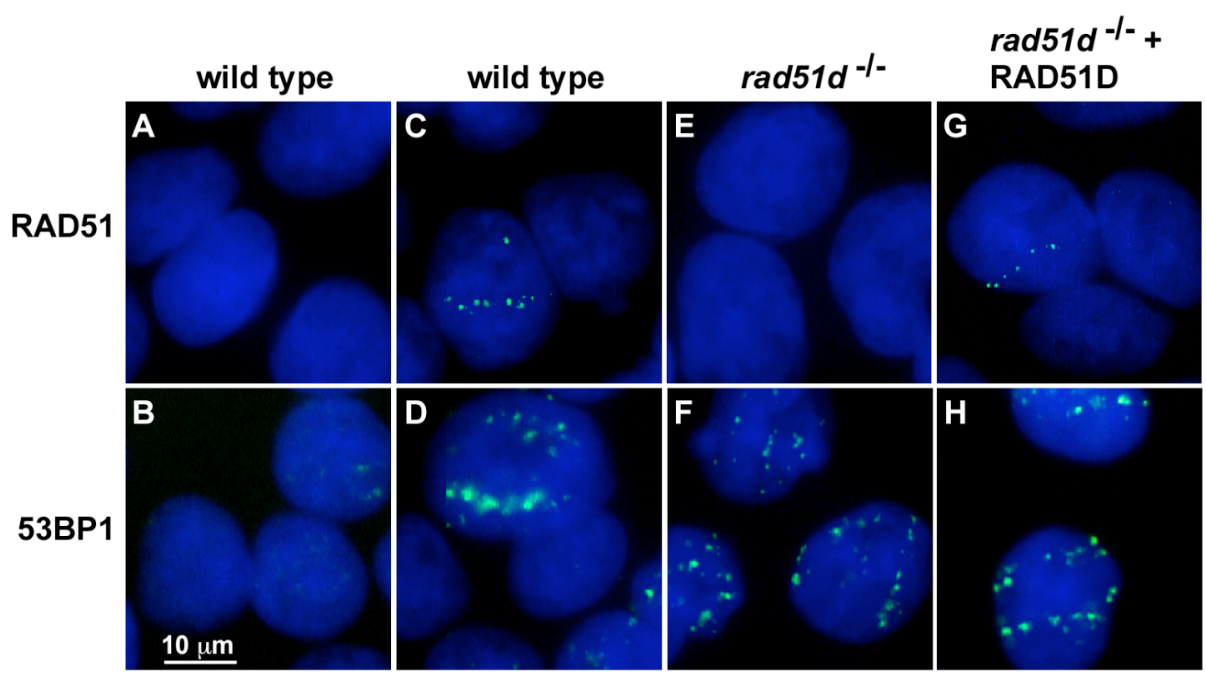


Figure 2

A

SiRNA
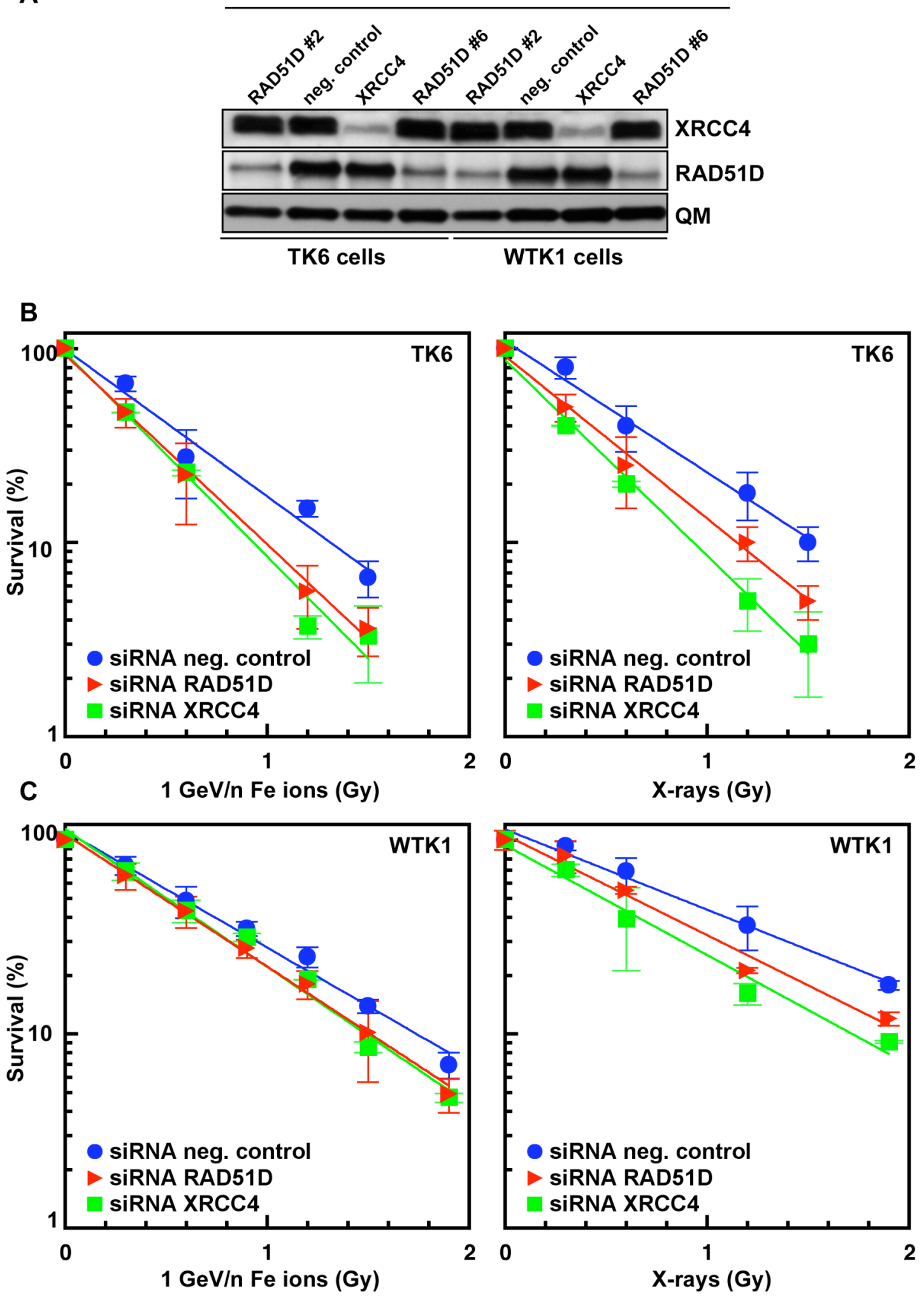
Figure 3
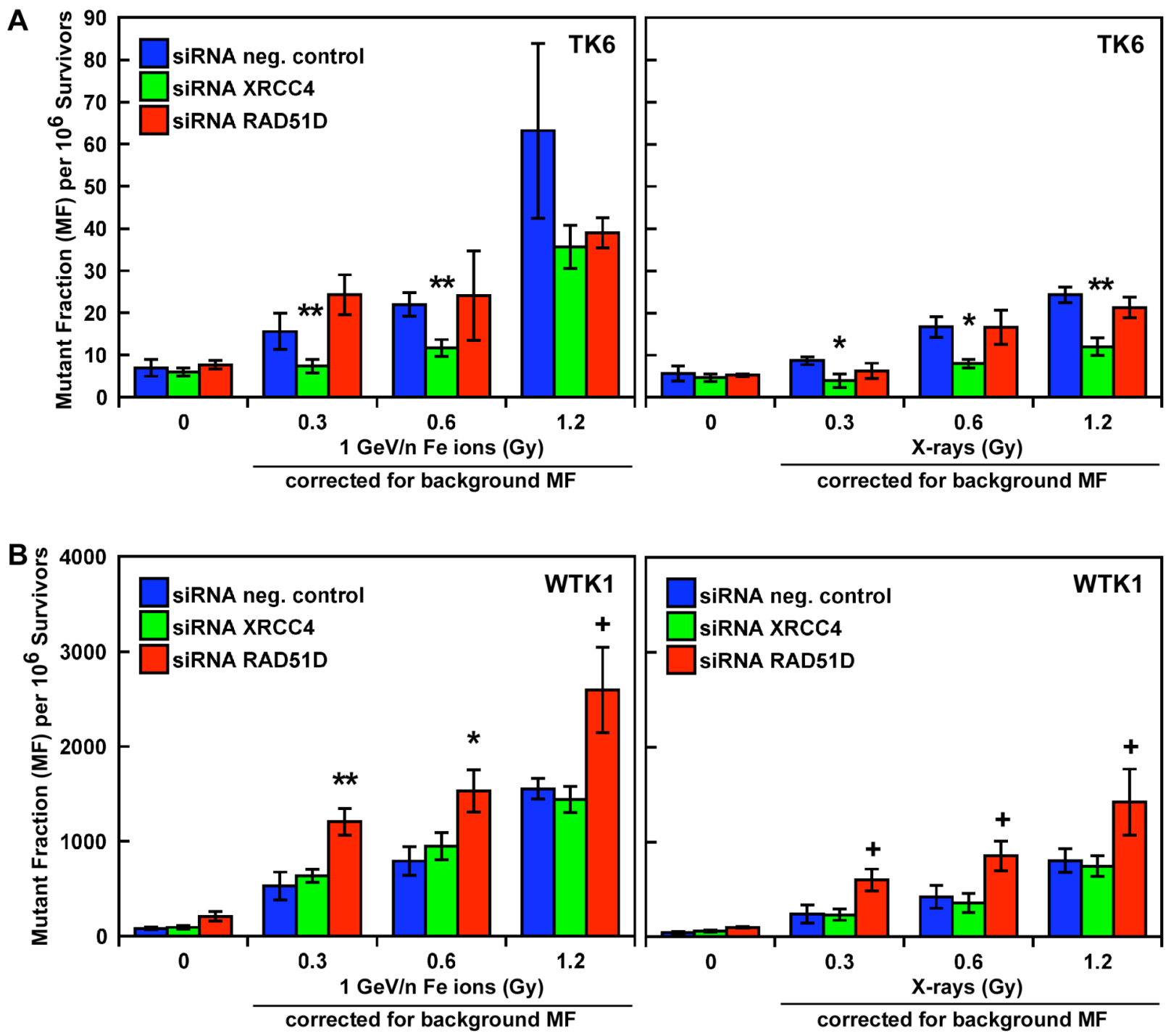
Figure 4

A

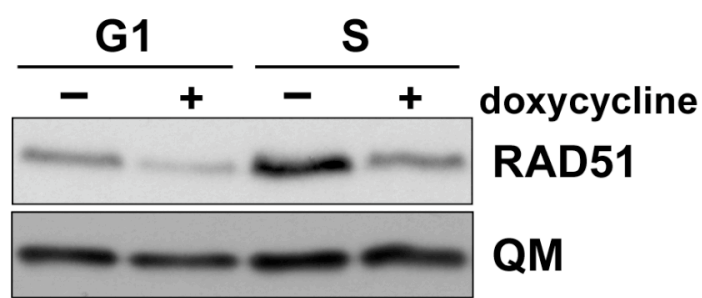

C

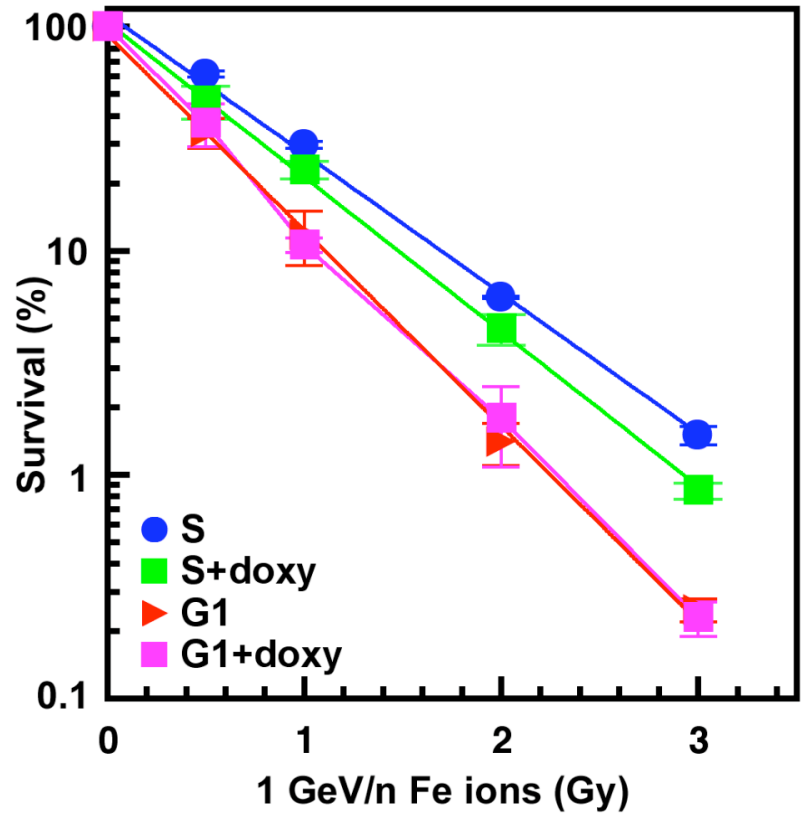

B

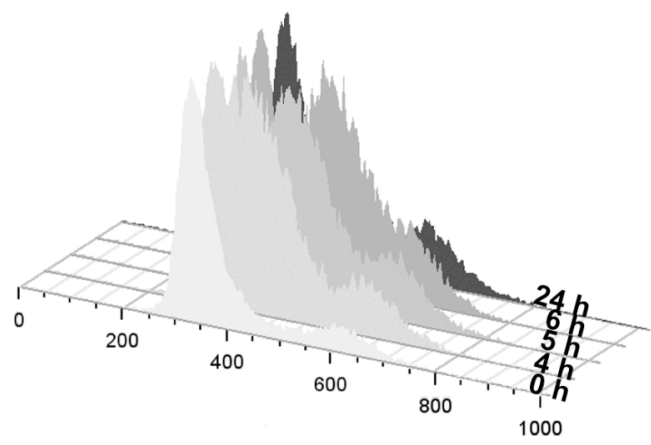

D

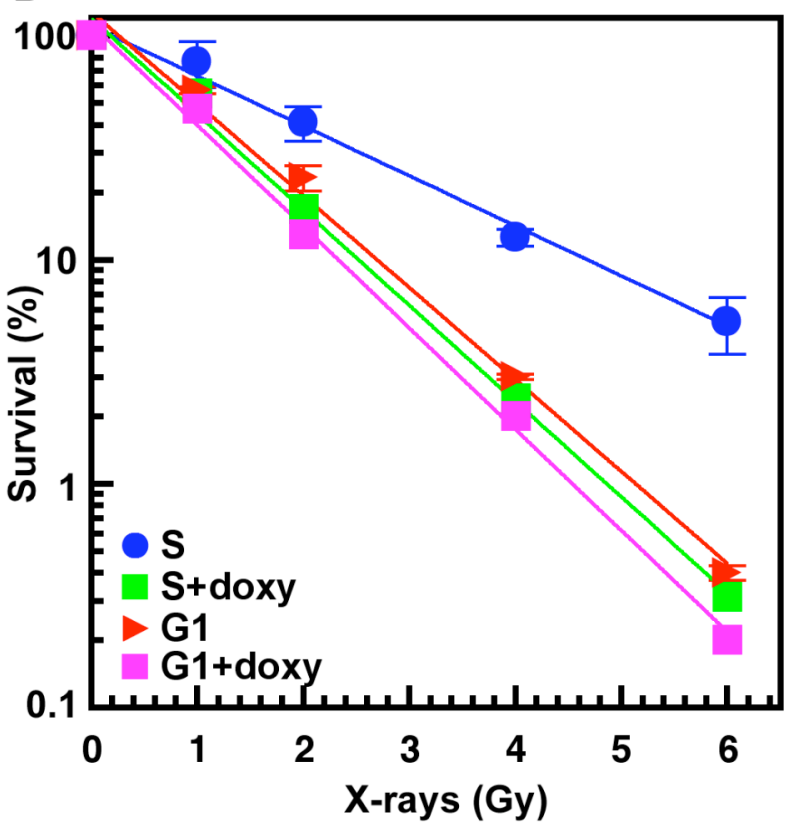


Supplementary Figure S1
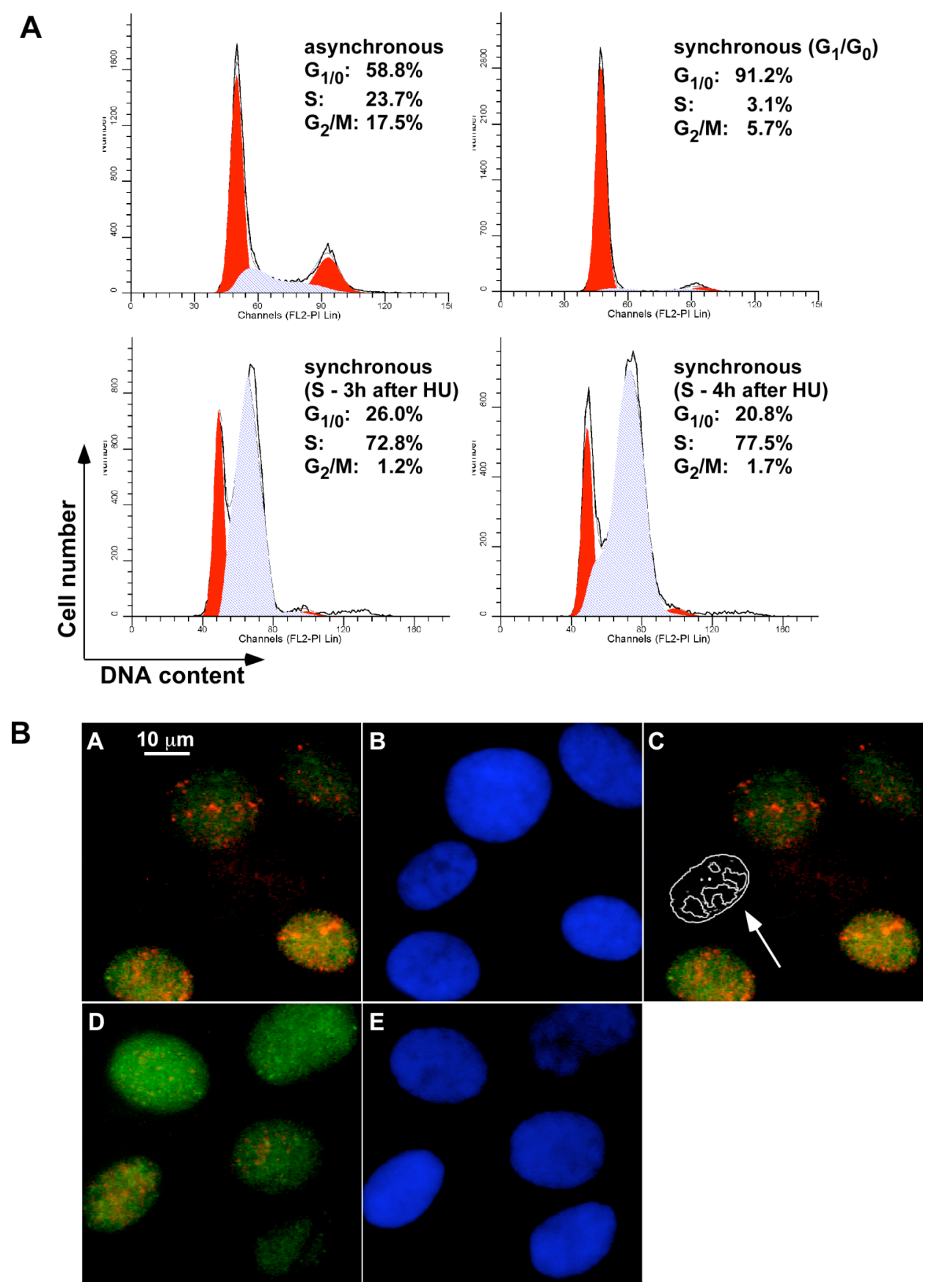
Supplementary Figure S2

A
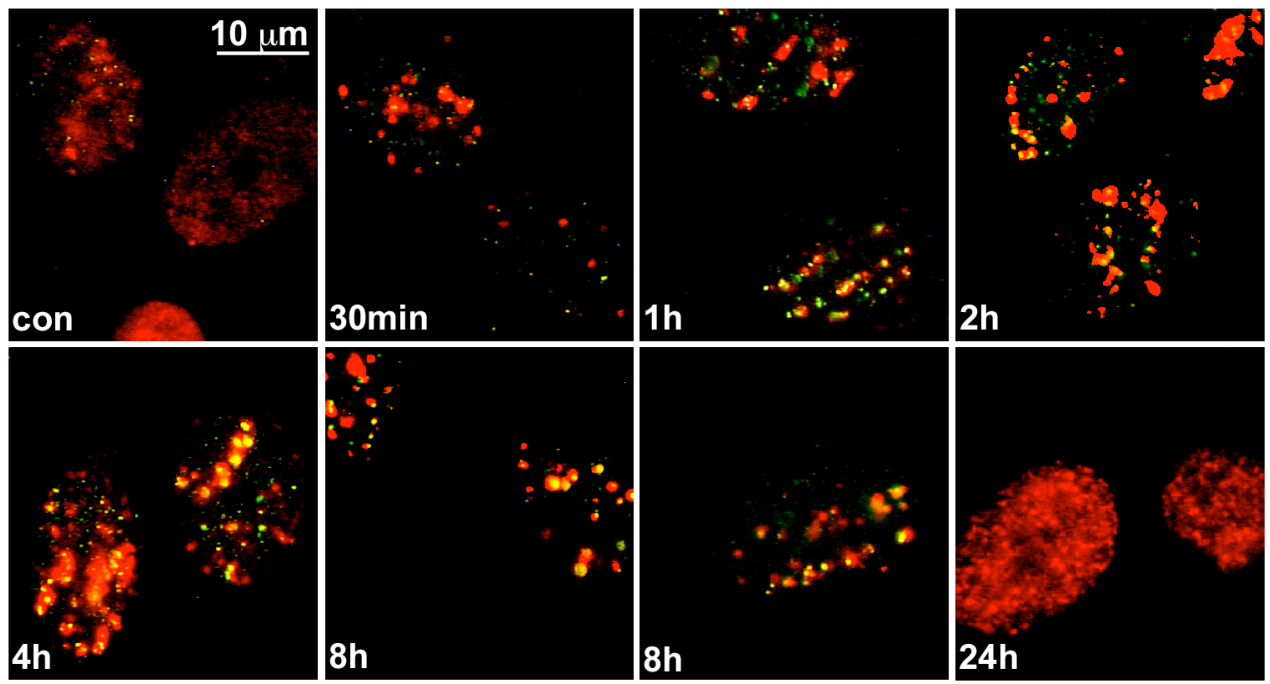

B

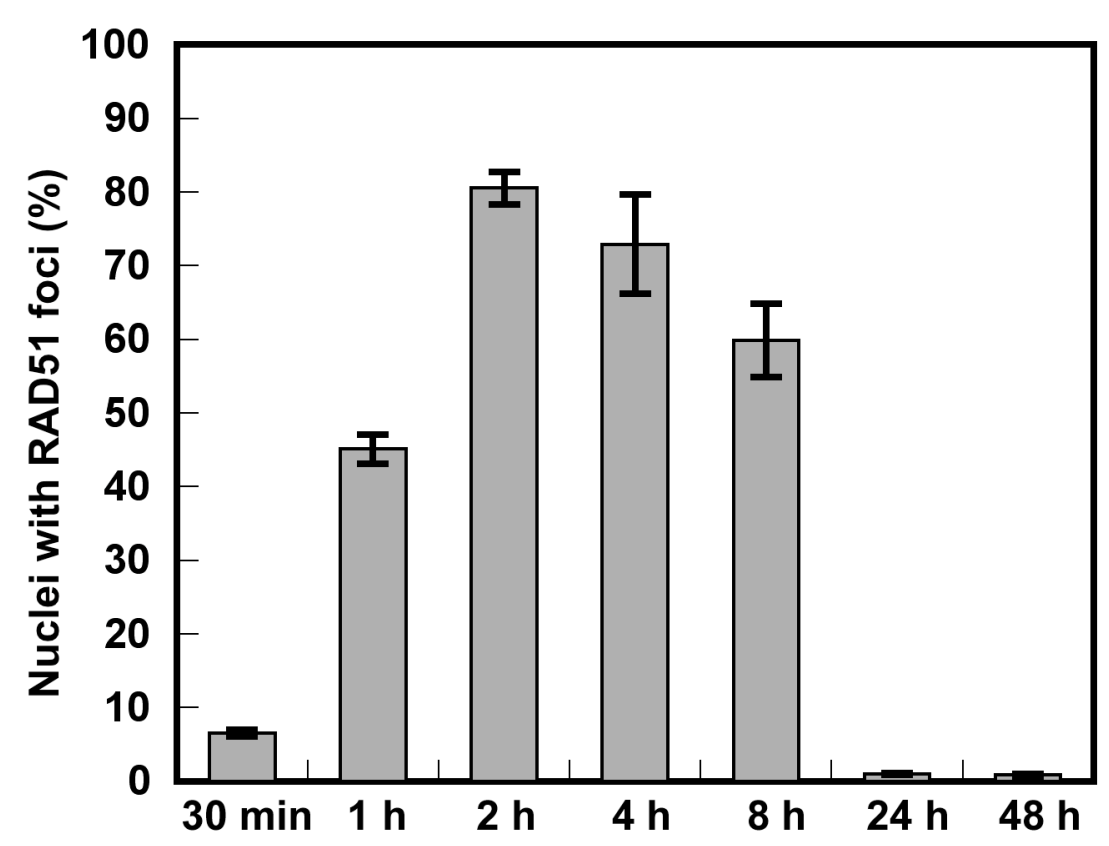

